\title{
On the relationship of polar mesospheric cloud ice water content, particle radius and mesospheric temperature and its use in multi-dimensional models
}

\author{
A. W. Merkel ${ }^{1}$, D. R. Marsh ${ }^{1}$, A. Gettelman ${ }^{1}$, and E. J. Jensen ${ }^{2}$ \\ ${ }^{1}$ National Center for Atmospheric Research, Boulder, Colorado, USA \\ ${ }^{2}$ NASA Ames Research Center, Moffett Field, California, USA
}

Received: 16 May 2009 - Published in Atmos. Chem. Phys. Discuss.: 3 July 2009

Revised: 8 October 2009 - Accepted: 9 November 2009 - Published: 24 November 2009

\begin{abstract}
The distribution of ice layers in the polar summer mesosphere (called polar mesospheric clouds or PMCs) is sensitive to background atmospheric conditions and therefore affected by global-scale dynamics. To investigate this coupling it is necessary to simulate the global distribution of PMCs within a 3-dimensional (3-D) model that couples large-scale dynamics with cloud microphysics. However, modeling PMC microphysics within 3-D global chemistry climate models (GCCM) is a challenge due to the high computational cost associated with particle following (Lagrangian) or sectional microphysical calculations. By characterizing the relationship between the PMC effective radius, ice water content $(i w c)$, and local temperature $(T)$ from an ensemble of simulations from the sectional microphysical model, the Community Aerosol and Radiation Model for Atmospheres (CARMA), we determined that these variables can be described by a robust empirical formula. The characterized relationship allows an estimate of an altitude distribution of PMC effective radius in terms of local temperature and $i w c$. For our purposes we use this formula to predict an effective radius as part of a bulk parameterization of PMC microphysics in a 3-D GCCM to simulate growth, sublimation and sedimentation of ice particles without keeping track of the time history of each ice particle size or particle size bin. This allows cost effective decadal scale PMC simulations in a 3-D GCCM to be performed. This approach produces realistic PMC simulations including estimates of the optical properties of PMCs. We validate the relationship with PMC data from the Solar Occultation for Ice Experiment (SOFIE).
\end{abstract}

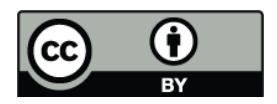

Correspondence to: A. W. Merkel (merkel@ucar.edu)

\section{Introduction}

The Aeronomy of Ice in the Mesosphere (AIM) satellite was launched in April 2007 with its primary focus to study the formation of Polar Mesospheric Clouds (PMC) (Russell et al., 2009). Several investigations based on the first season of PMC observations have shown complex dynamical features, evidence of small wavelength gravity wave activity and planetary wave activity (Rusch et al., 2009; Chandran et al., 2009; Merkel et al., 2009). These studies indicate that seasonal variability of PMCs is largely affected by the dynamical conditions of the summer atmosphere. In addition, there is evidence of a long-term increase and solar-cycle variation in PMC brightness and frequency (Deland et al., 2007; Shettle et al., 2009). Long-term PMC variability is thought to be an indicator of a changing climate (Thomas et al., 2003), however satellite observations of PMCs are limited to about three solar cycles (Deland et al., 2007). Therefore modeling efforts are crucial in understanding new AIM PMC observations in the context of longer-term changes resulting from solar and anthropogenic forcing.

Modeling efforts that use a Lagrangian ice transport scheme in a 3-D middle atmosphere model produce detailed information about cloud processes and properties on seasonal time-scales (Berger and Lübken, 2006; Lübken and Berger, 2007). However, Lagrangian models are inefficient for longterm integrations (such as several decades) due to inherent computational cost and data storage limitations. Although not as computational exhaustive, models that use sectional codes to include ice microphysics are also computationally expensive (Bardeen et al., 2008, 2009). For instance, the Bardeen et al. (2009) PMC ice model includes 28 dust bins and 28 ice particle bins with each advected individually at each time step. To overcome this problem, it is desirable to

Published by Copernicus Publications on behalf of the European Geosciences Union. 
have a bulk parameterization for PMC microphysics to include in multi-dimensional atmospheric global models to facilitate simulations on decadal time scales.

A requirement for an accurate bulk PMC parameterization is a reasonable estimate of the mean ice mass, which depends on particle growth rates. The growth of ice particles not only depends on the saturation conditions and temperature but on the size of the particle. Therefore, given an estimate of effective radius, the microphysical equations for cloud particle growth, sublimation and sedimentation (Gadsden et al., 1998) can be used to model PMCs within a 3-D global chemistry climate model (GCCM). Siskind et al. (2006) introduced a bulk PMC parameterization into the CHEM2D model to study the radiative feedback from PMCs. Their simplistic parameterization assumes a constant particle size of $70 \mathrm{~nm}$. This type of assumption will cause errors in the amount of mean ice mass created, destroyed and sedimented at each time step, since the microphysics is calculated for only one particle size. To improve upon Siskind et al. work, we developed a method to estimate the effective radius at each time step.

By analyzing the relationship between the PMC effective radius in $\mathrm{nm}$, ice water content $(i w c)$ in $\mathrm{g} / \mathrm{cm}^{3}$, and local temperature $(T)$ in Kelvin, we determined that these variables can be described by a robust empirical formula. For our purposes we use this relationship as part of a bulk parameterization of PMCs in a GCCM. However, this relationship has the potential to be used in other applications to characterize PMCs. This paper will illustrate this relationship and its potential use in multi-dimensional global climate models.

\section{Relationship between PMC, iwc, $T$, and radius}

Our work is based on the development of a similar relationship found between temperature, ice water content and particle radius for tropospheric cirrus clouds (Boudala et al., 2002). Boudala et al. developed an empirical formula using in-situ cloud data to predict effective radius of tropospheric ice particles using local $T$ and $i w c$ in a global model. We adopted this empirical formula to characterize the relationship between these variables for PMCs, using an ensemble of simulations from the one-dimensional version of the Community Aerosol and Radiation Model for Atmospheres (CARMA) microphysical model (Rapp and Thomas, 2006). To be consistent with the empirical formula as defined for tropospheric data, we are using the term $i w c$ as a measure of the ice mass density in units of $\mathrm{g} / \mathrm{cm}^{3}$. The CARMA model is used to establish this relationship because it provides full microphysical processes including nucleation, condensational growth, particle sedimentation and transport of ice particles on small time scales. The CARMA model handles three interactive constituents: meteoric smoke particles (distribution by Hunten et al., 1980), ice particles and mesospheric water vapor. The model is comprised of 120 altitude levels from 72 to $102 \mathrm{~km}$ and consists of 40 logarithmically spaced radius size bins $\left(r_{i}\right)$ for ice particles ranging between 2 and $900 \mathrm{~nm}$. The model timestep is $100 \mathrm{~s}$. Ice and water vapor within CARMA is transported by the mean vertical wind, eddy diffusion and particle sedimentation.

For this work, we use the same assumptions and settings as described for the CARMA reference case in Rapp and Thomas (2006), except our method differs in the specification of the background temperature profile. The reference case as described in Rapp and Thomas, forces the temperature altitude profile to be constant throughout a 48-h simulation. Their simulation did not take into account the changing background environment that occurs on timescales shorter than 48-h. To simulate the dynamical variability of the background temperature and the effects of changing temperatures on PMC ice particles, we used temperature time histories from simulations from the 3-D coupled chemistry-climate Whole-Atmosphere Community Climate Model (WACCM) (Garcia et al., 2007) as input to the CARMA model. In effect, our CARMA simulations were updated with a new temperature altitude profile at each timestep. The temperature histories were obtained by recording a high latitude mesosphere air parcel's movement over 4-days from a WACCM simulation. A temperature altitude profile (associated with the location of the air parcel) at each WACCM time-step was recorded. Because CARMA is a 1-D model we chose to ignore wind shear in the temperature altitude profiles and assumed all altitudes moved together with the air parcel. We compiled fifteen 4-day temperature histories from WACCM output over 1 July-5 July and used them as input to CARMA. A WACCM simulation over 4-days was used to capture several different dynamical timescales and to encompass particle development under hydrated and dehydrated conditions. It is noted that subgrid-scale gravity waves are not included in the simulations. The water vapor profile to initialize each CARMA run was obtained from the first time step of each WACCM time history. We note that the history curtains were obtained from a version of WACCM that does not contain PMC microphysics or dehydration in the mesosphere region. Therefore all the initial water vapor profiles are hydrated. Figure 1 shows the geographic trajectories that make up the WACCM temperature time histories used in this study. Each time history has a different geographic start point indicated by the diamonds in the figure. By using temperature histories as input to CARMA, we have indirectly simulated the changing background temperature due to atmospheric dynamics. To ensure that we modeled conditions typical of a PMC environment and to fill the entire parameter space of relevance to PMCs, we chose temperature trajectories that represent observed polar mesospheric summer temperatures with typical variability. The temperature range of the trajectories between $80 \mathrm{~km}$ and $90 \mathrm{~km}$ is $115 \mathrm{~K}$ to $160 \mathrm{~K}$ in this four-day period. We found that 1-D CARMA should not be initiated with supersaturated conditions (the consequence is too many nucleated particles in the first time step). Therefore we chose 


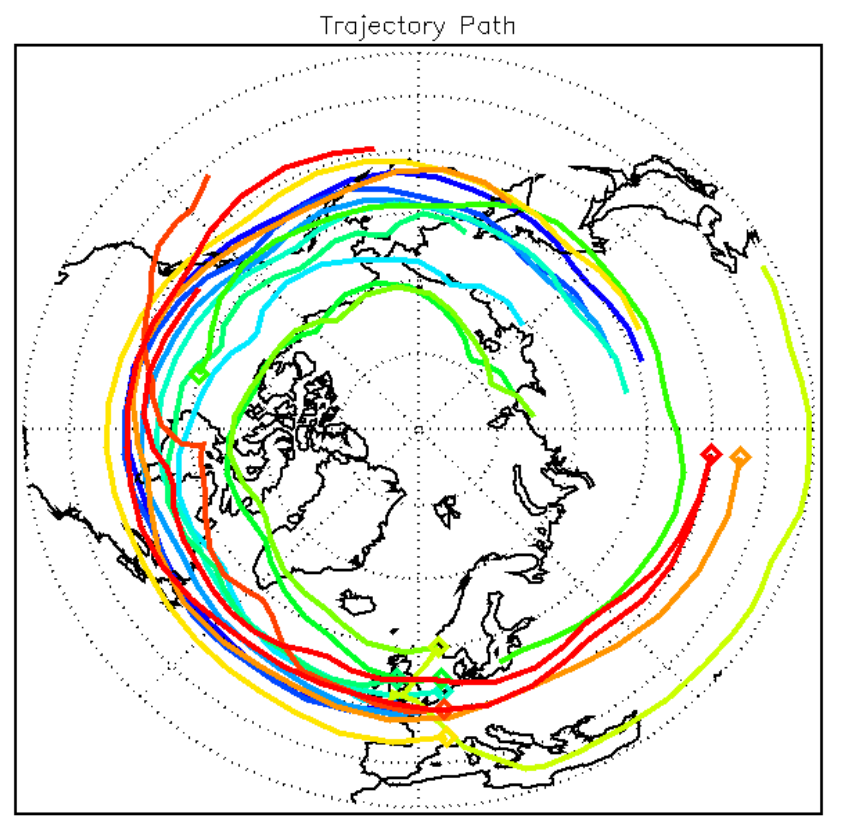

Fig. 1. Trajectory locations from WACCM simulations used in CARMA simulations. The diamond indicates the starting point of each of 15 trajectories. Trajectories are initialized on 1 July and run for 4 days.

trajectories that naturally start with warmer temperatures and then moved to colder saturated regions. This criteria, in effect, eliminated the use of all trajectories at the high polar latitudes. However, since CARMA only responds to the input variables at each time step, what matters is the realism and range of the input temperature profiles not the geographic location of the trajectory.

An example of a temperature history for one WACCM trajectory used in a CARMA simulation is shown in Fig. 2a. The temperature altitude profile varies at each CARMA timestep. The input temperature profiles are typical of mesosphere temperatures and vary between $115 \mathrm{~K}$ to $160 \mathrm{~K}$ between 80 and $90 \mathrm{~km}$. The trajectory start point was $105^{\circ} \mathrm{W}$ longitude $60^{\circ} \mathrm{N}$ latitude (green diamond in Fig. 1). Figure $2 \mathrm{~b}$ shows the resulting total $i w c$ calculated in CARMA for the temperature input shown in Fig. 2a. Figure $2 \mathrm{c}$ and d show the evolution of water vapor and effective radius. As mentioned, the first time step is forced with a hydrated water vapor profile from WACCM. In each subsequent time step, water vapor is a free variable that is modified by the microphysics, as shown in the Fig. 2c. CARMA provides a discrete number distribution $n_{i}\left(r_{i}\right)$ for the 40 logarithmically spaced radius size bins $\left(r_{i}\right)$ ranging between 2 and $900 \mathrm{~nm}$. We use this information to calculate an area-weighted effective radius $\left(r_{\text {eff }}\right)$ (Wyser, 1998) at each time step and altitude level using Eq. (1). The result is plotted in Fig. 2d.

$r_{\mathrm{eff}}=\frac{\sum r_{i}^{3} n_{i}}{\sum r_{i}^{2} n_{i}}$
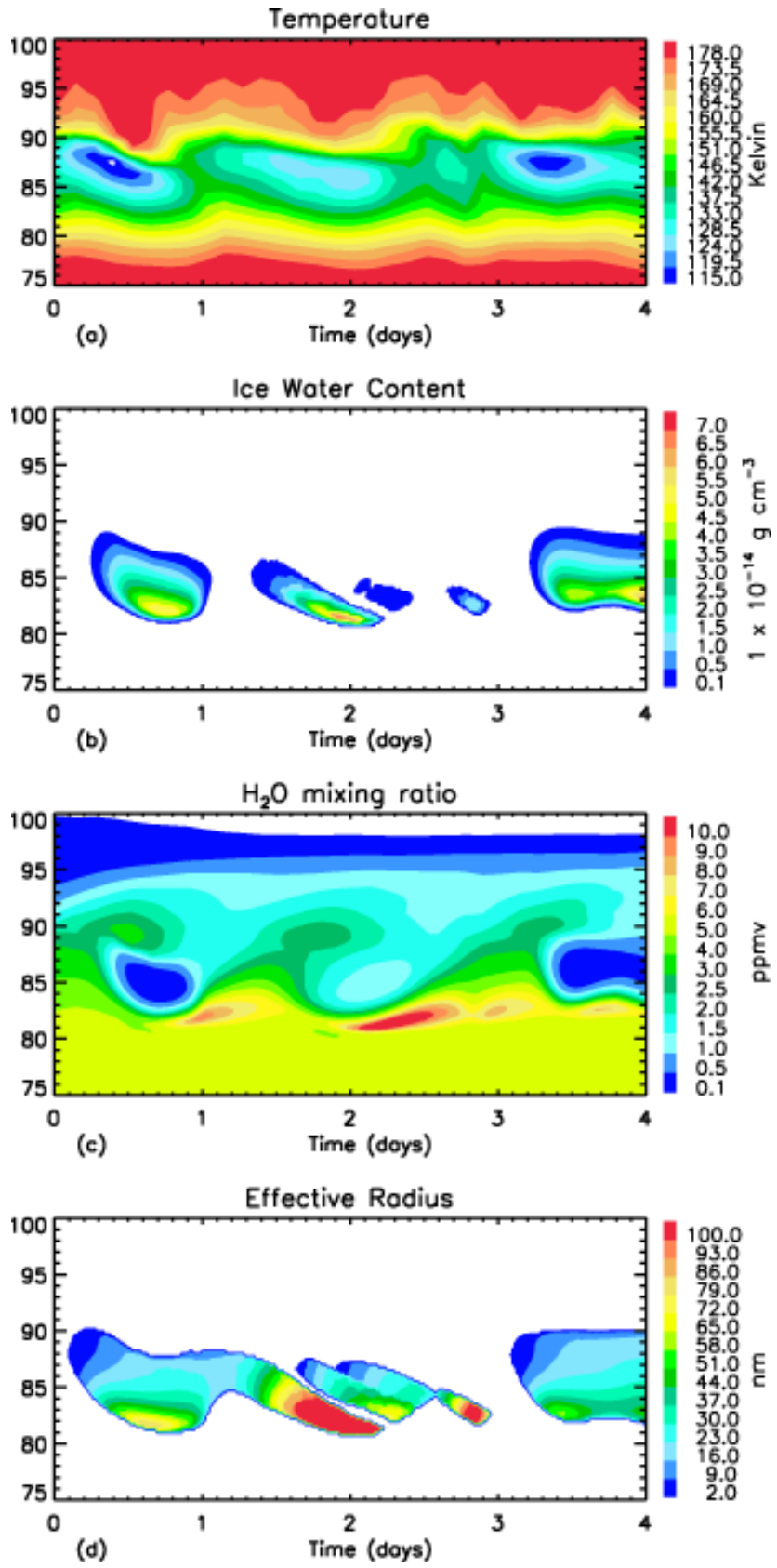

Fig. 2. (a) Temperature time history from a WACCM trajectory simulation based on a trajectory starting from $105^{\circ} \mathrm{W}$ and $60^{\circ} \mathrm{N}(\mathbf{b})$ ice water content from the CARMA simulation associated with the temperature input in the first panel. (c) Evolution of water vapor, the first time step is forced with a water vapor profile from WACCM. (d) Evolution of effective radius.

This expression for $r_{\mathrm{eff}}$ is independent of a specific observing technique and is different than the effective optical radius used by Karlsson and Rapp (2006). The latter is a measure of the optically dominant particle size, which depends on the wavelength and scattering angle of observation. We use an area-weighted radius without an observing bias to better 

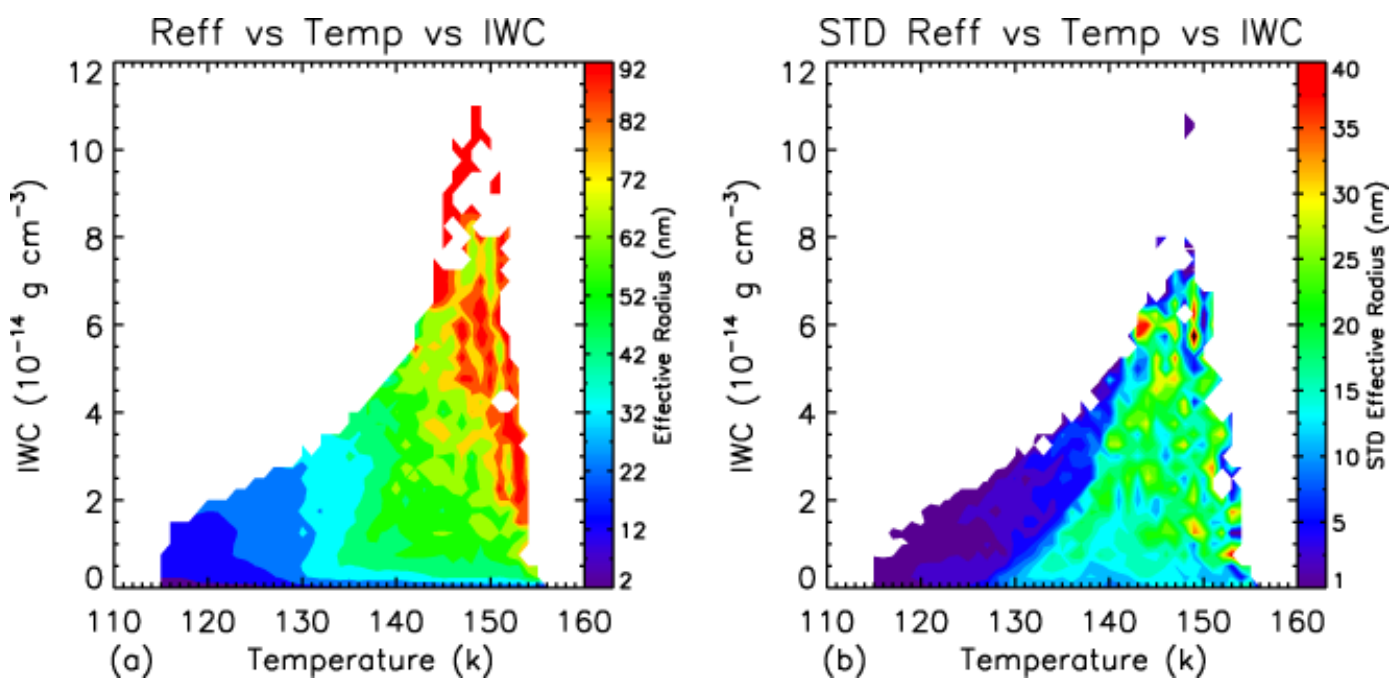

Fig. 3. Surface plots of the relationship between $r_{\text {eff }}, i w c$ and $T$. (a) $r_{\text {eff }}$ calculated directly from the particle distribution at each point in the CARMA history files. (b) standard deviation of $r_{\text {eff }}$ in each $T$ and $i w c$ bin.

represent the growth, sedimentation and sublimation of the particle, since these processes depend on the particle area.

The four panels in Fig. 2 show how the distribution of ice water content, water vapor and particle size varies with the background temperature. The ice grows, sublimates and reappears as the temperature varies. This is a more realistic representation of the ice water distribution over altitude and time than the reference CARMA simulation presented in Rapp and Thomas (2006) where the model was initialized with supersaturated conditions and let to run for $48 \mathrm{~h}$ without changing the temperature profile. An additional advantage to including a changing temperature environment is better coverage of the parameter space of $T, i w c$ and the distribution of particle sizes for defining a relationship between these variables.

From the ensemble of CARMA simulations, approximately 75000 predicted $i w c, T$ and $r_{\text {eff }}$ combinations were extracted using data at all relevant PMC altitudes. To illustrate the relationship between $r_{\mathrm{eff}}, i w c$ and $T$ compiled from the simulations, a surface plot of $r_{\text {eff }}$ versus $i w c$ and $T$ is shown in Fig. 3a. Figure 3b shows the standard deviation (STD) of $r_{\text {eff }}$ in each $T$ and $i w c$ bin from Fig. 3a. Figure 3a demonstrates that for a given $i w c$, effective radius increases with increasing temperature. This is inherent from the relationship of the background temperature with the ice particle distribution at any particular altitude. The x-axis (temperature) in Fig. 3a and b can be thought of as a proxy for altitude. Temperature is coldest near the mesopause corresponding to an altitude where the ice particles are in infancy. This altitude region relates to the lower left part of Fig. 3a and b, where the particles are generally small and have a small total $i w c$. The figures indicate that at very cold temperatures (like near the mesopause) the PMC particles are small with very little variation. As the particles mature they fall to lower altitudes where they encounter warmer temperatures. This altitude region relates to the right part of Fig. $3 \mathrm{a}$ and $\mathrm{b}$. The particles sizes in this area are well mixed and include larger radii with a stable standard deviation near $15-20 \mathrm{~nm}$. At any specific temperature (altitude) there is a mix of particles sizes. By knowing both the $T$ and $i w c$ the particle size can be estimated. For example, if a line is drawn vertically at $150 \mathrm{~K}$ in Fig. 3a, several different groups of radii are intersected. Each radii group can be described by the $i w c$ at a specific temperature. Using all altitude combinations of $T, i w c$, and $r_{\text {eff }}$ (where $i w c>0$ ), a least-squares fit of the model data to the following empirical formula (Boudala et al., 2002) was calculated:

$r_{\text {eff } p}=p_{1} i w c^{p_{2}} e^{\left(p_{3} T\right)}$

where $p_{1}=760.34, p_{2}=0.235$ and $p_{3}=0.034, r_{\text {eff }}$ in units of $\mathrm{nm}, i w c$ is in units of $\mathrm{g} / \mathrm{cm}^{3}$ and $T$ in Kelvin $(\mathrm{K})$. Then, using $i w c$ and $T$ pairs from the CARMA simulations and Eq. (2), a parameterized effective radius $\left(r_{\text {eff }} p\right)$ can be calculated. Figure 4 illustrates the resulting surface plot of $r_{\text {eff }}$ versus $i w c$ and $T$. It is clear that the empirical formula in Eq. (2) reproduces the relationship shown in Fig. 3a. Sensitivity tests were performed to characterize the stability of the empirical. We ran simulations with the mesopause altitude displaced by $2 \mathrm{~km}$, and CARMA initialized with a dehydrated water vapor profile. In addition, we reran all trajectories through CARMA using the Bardeen et al. (2008) dust distribution in place of the Hunten et al. (1980) distribution. In all cases the fit parameters did not change by more than 5\%. A comparison of a full season of WACCM/CARMA data shows a very similar relationship of these variables to that shown in Fig. 3a.

Figure 5 illustrates the distribution of effective radius from the CARMA simulations. The black line represents the 


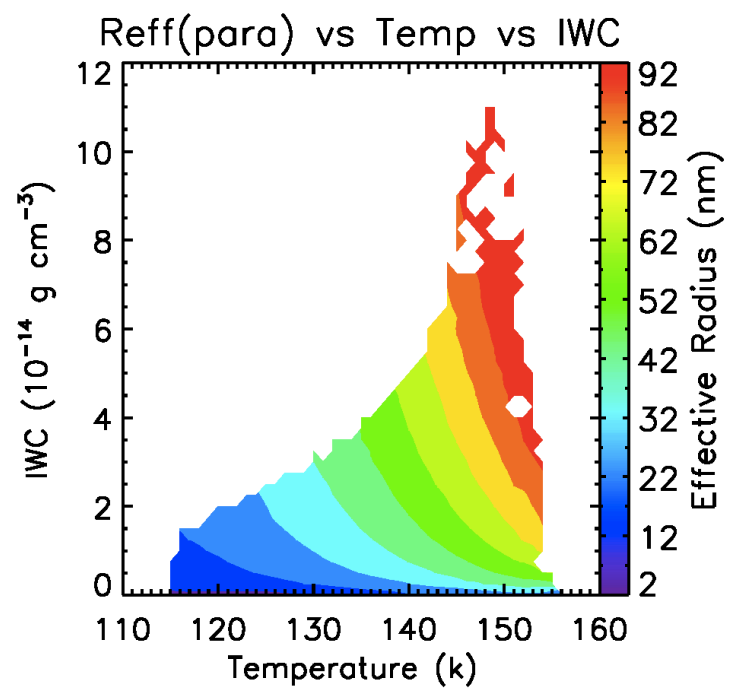

Fig. 4. Surface plot of the relationship between $r_{\text {effp }}$, iwc and $T$. $r_{\text {eff } p}$ is calculated from the parameterization illustrated in Eq. (2).

distribution of all effective radii at all altitudes calculated from the ensemble of CARMA simulations using WACCM trajectories and Eq. (1). The blue line represents the parameterized effective radius calculated using all combinations of $i w c>0$ and $T$ pairs from the same CARMA simulations and Eq. (2). The empirical formula reproduces the overall distribution of effective radii, which peaks near $20 \mathrm{~nm}$.

\section{Validation of the parameterized effective radius}

\subsection{CARMA comparisons}

In this section we compare several properties of PMCs calculated as part of the ensemble of CARMA simulations to those calculated using the parameterized effective radius. Figures 6,7 , and 8 show PMC effective radius, number density and PMC optical properties as output from three of the CARMA simulations and those derived using the empirical formula described in Sect. 2. Values in Fig. 6 correspond to the temperature history and iwc shown in Fig. 2. Figure $6 \mathrm{a}$ shows the evolution of effective radius as shown in Fig. 2d. In comparison, the top right panel (Fig. 6b) illustrates the parameterized effective radius calculated with the empirical formula in Eq. (2) using the local WACCM $T$ and $i w c$ at each grid point in altitude and time from the panels in Fig. 2. While the error of the parameterized effective radius in Fig. 6b at any specific location in time and altitude could be rather large (in comparison to Fig. 6a), there is a striking similarity of the altitude and time distribution of the effective radius, indicating that a expression that takes local temperature and ice water content into account, adequately models the vertical distribution of the area-weighted radius at each time step. It is this important product of the empirical

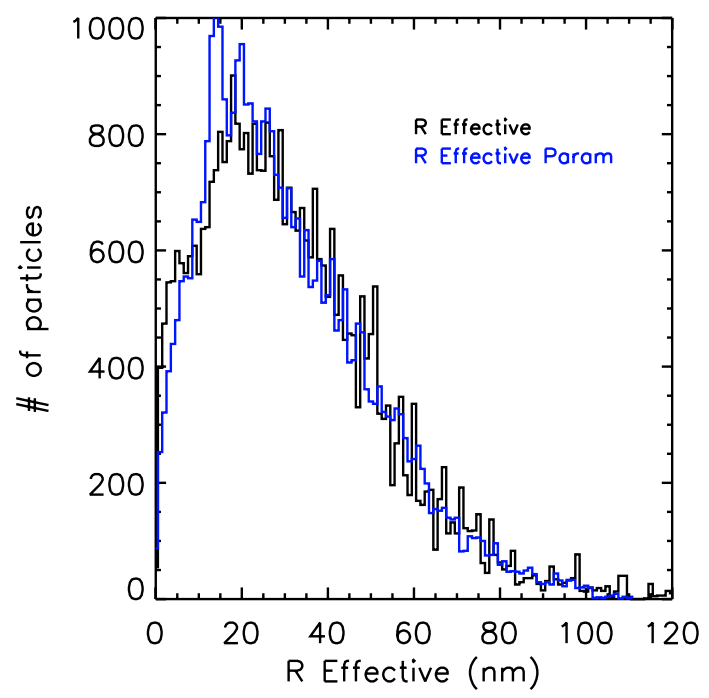

Fig. 5. Histogram of the distribution of $r_{\text {eff }}$ calculated at each point from the particle distribution in CARMA. The blue curve illustrates the particle distribution of $r_{\mathrm{eff}} p$ calculated with the parameterization.

formula, to adequately estimate the vertical distribution of ice particle size at each horizontal grid point, that allows for the effective application of microphysical processes (growth, sublimation and sedimentation) to parameterized effective radius in a global model. Figures $7 \mathrm{a}-\mathrm{d}$ and $8 \mathrm{a}-\mathrm{d}$ represent the temperature history of $i w c, r_{\text {eff }}$ and $r_{\text {eff }} p$ for two additional CARMA run examples, each with a dimmer PMC than the previous example. Figure 7 trajectory has a start point at $5^{\circ} \mathrm{E}$ longitude and $53^{\circ} \mathrm{N}$ latitude. Figure 8 trajectory has a start point at $5^{\circ} \mathrm{W}$ longitude and $55^{\circ} \mathrm{N}$ latitude. Again, the altitude and time distribution of the parameterized effective radius is in good agreement with the simulated distribution. The empirical formula is able to describe the characteristics of PMC particle growth in terms of $T$ and $i w c$, without needing to know the history of the ice particle. For example, in Fig. 8, a cloud is formed between the simulated days 2 and 3. By using the local temperature structure and $i w c$ at each timestep in this time range, the parameterization recreates the cloud formation around $88 \mathrm{~km}$ and the development of the cloud as the particles grow larger and move to lower altitudes.

The number density is a useful diagnostic variable that allows estimation of the observed PMC radiance to compare models to satellite observables. Given a parameterized effective radius and ice water content, it is a simple task to calculate the ice particle number density $\left(N_{\text {para }}\right)$ assuming the particles are spherical:

$N_{\text {para }}=\frac{i w c}{\frac{4}{3} \pi r_{\text {eff }}^{3} \rho_{\text {ice }}}$

where $\rho_{\text {ice }}$ is the density of ice $\left(0.93 \mathrm{~g} \mathrm{~cm}^{-3}\right)$. The derived 

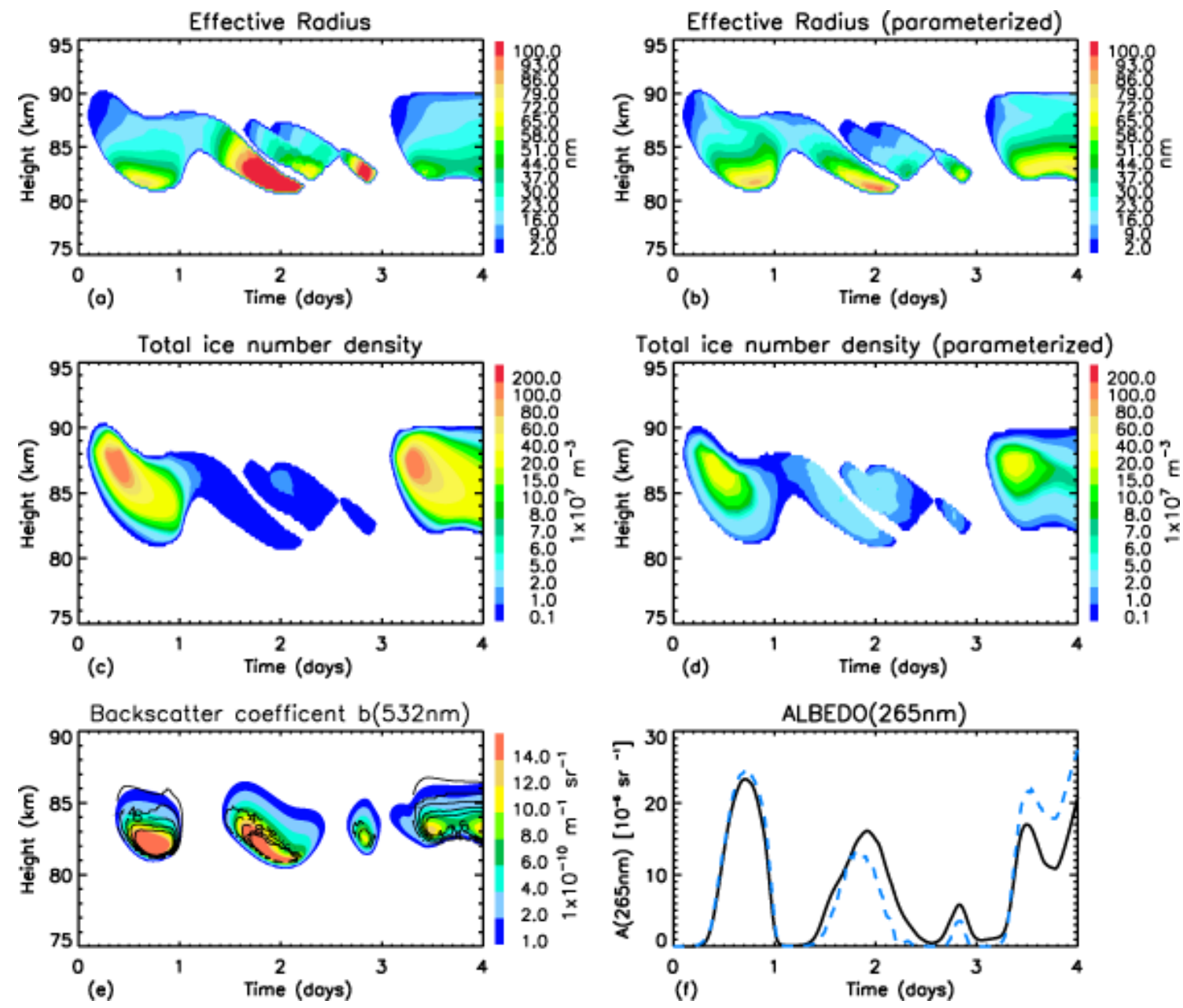

Fig. 6. Panel plots illustrating the effectiveness of the parameterization. The panels correspond to the simulation shown in Fig. 2. The left column illustrates cloud properties calculated from the output of the CARMA simulation with full microphysics and particle distribution. The right column illustrates properties calculated with the parameterized effective radius. The bottom two panels illustrate PMC optical signals. The signal calculated directly from the CARMA simulations are illustrated by the filled contour levels in the backscatter plot and by the solid black line in the albedo plot. Overplotted are $\beta$ and $A$ calculated using the parameterized effective radius and number density (contour lines in the backscatter plot and dashed blue line in the albedo plot).

number density can be compared to that calculated directly from the CARMA simulations (Figs. 6c, 7e and 8e). While $N_{\text {para }}$, shown in Figs. $6 \mathrm{~d}, 7 \mathrm{f}$ and $8 \mathrm{f}$ are not exactly the same as the number density that comes directly out of CARMA, the vertical distribution and time histories are very similar. Since the applied empirical formula provides a vertical distribution of particle size, the optical properties of PMCs such as backscatter coefficient $(\beta)$ and albedo $(A)$ can be easily calculated using Eqs. (4) and (5),

$\beta=\int_{0}^{\infty} \frac{d \sigma}{d \Omega}(r, \lambda, \vartheta) \cdot \frac{d N}{d r} \cdot d r$

and

$$
A=\int_{0}^{\infty} \int_{0}^{\infty} \frac{d \sigma}{d \Omega}(r, \lambda, \vartheta) \cdot \frac{d N}{d r} \cdot d r \cdot d z
$$

where $d \sigma / d \Omega$ is the Mie scattering cross-section derived applying standard Mie-scattering algorithms (Bohren and Huffman, 1983) and $\lambda$ and $\vartheta$ are the wavelength and scattering angle under consideration. The PMC vertical backscatter coefficient at $532 \mathrm{~nm}$ and nadir viewing PMC albedo at $265 \mathrm{~nm}$ are shown in Figs. 6e-f, $7 \mathrm{~g}-\mathrm{h}$ and $8 \mathrm{~g}-\mathrm{h}$. The "true" optical signals can be calculated directly from the discrete particle size distribution that comes directly from the CARMA simulations. The "true" optical signals are illustrated by the filled contour levels in the backscatter plots and by the solid black line in the albedo plots. Overplotted are $\beta$ and $A$ calculated using the parameterized effective radius and number density 

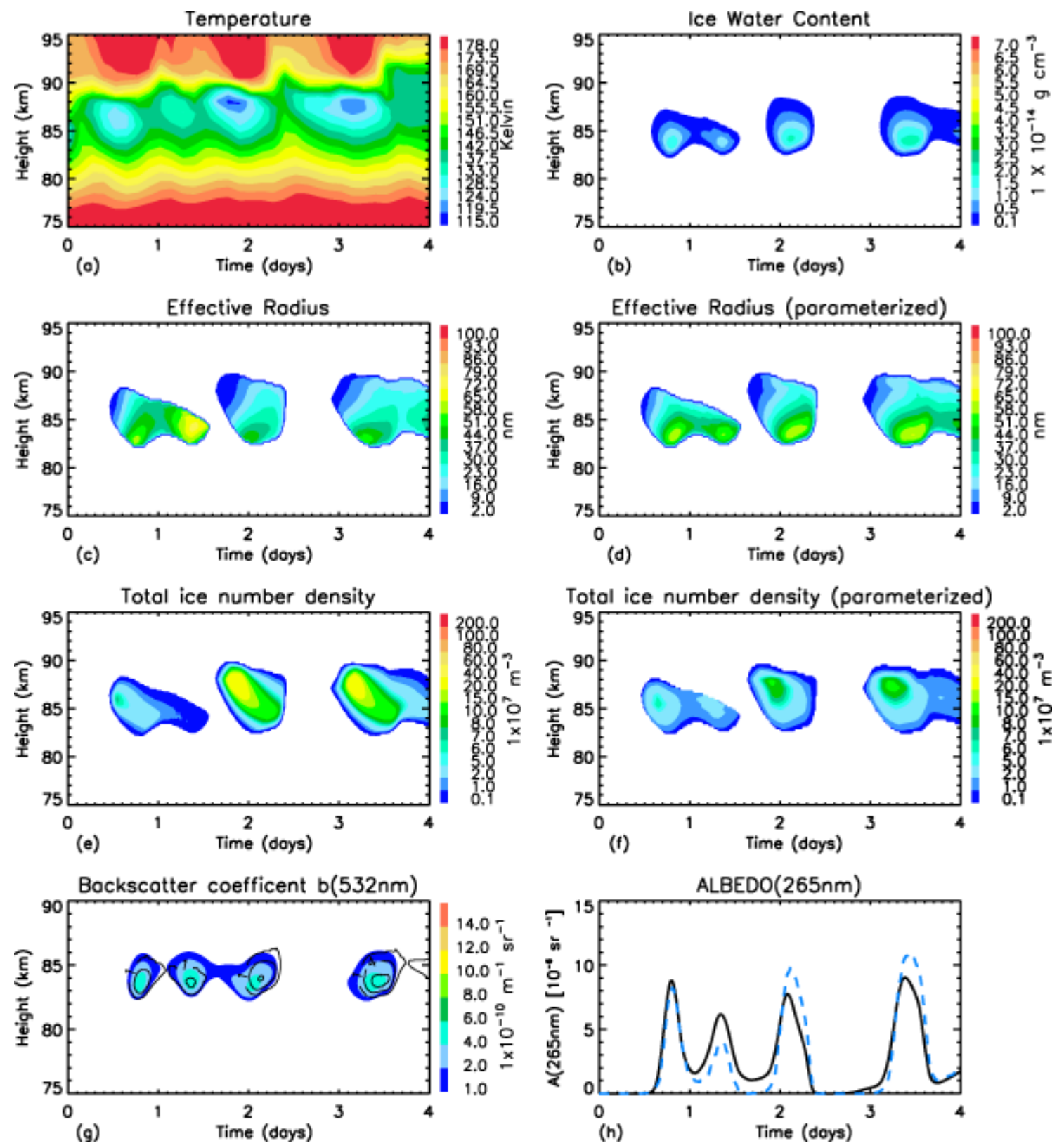

Fig. 7. Same as Fig. 6 but an alternate trajectory that has a start point at $5^{\circ} \mathrm{E}$ longitude and $53^{\circ} \mathrm{N}$ latitude. The top two panels illustrate the temperature and $i w c$ history.

(contour lines in the backscatter plot and dashed blue line in the albedo plot). One minor draw back to estimating the optical properties ( $\beta$ and $A$ ) using the parameterized $r_{\text {eff } p}$ and $N_{\text {para }}$, is that the particle size distribution $(d N / d r)$ must be assumed. From an analysis of the particle size distributions that come out of the detailed CARMA simulations and using the width as a tuning parameter we determined that a Gaussian distribution (Eq. 6) with a $12 \mathrm{~nm}$ width $(\Delta r)$ recreates the "true" optical properties. $\frac{d N}{d r}=\frac{N_{\text {para }}}{\sqrt{2 \pi} \Delta r} \exp \left(-\frac{\left(r-\left\langle r_{\text {mean }}\right\rangle\right)^{2}}{2 \Delta r^{2}}\right)$

An error is introduced by using $r_{\text {eff }}$ (area-weighted parameterized radius) instead of a number weighted mean radius to calculate backscatter and albedo. To minimize this error, an estimated mean radius is calculated as a function of $r_{\text {eff }}$ from the ensemble of CARMA simulations. Using linear regression we calculated $r_{\text {mean }}=0.9037 r_{\text {eff }}-3.18 \mathrm{~nm}$, and use it in the calculation in Eq. (6). 

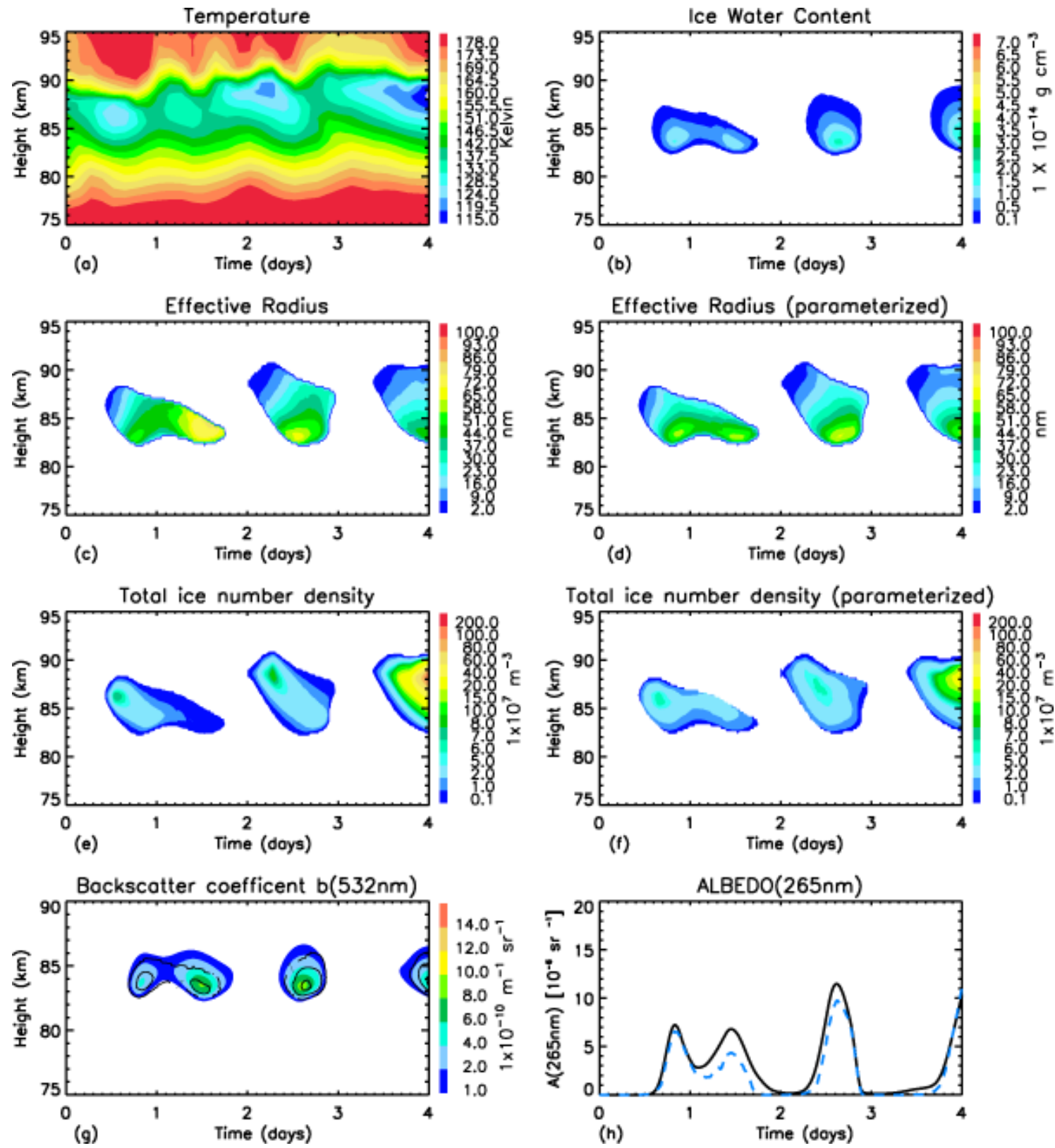

Fig. 8. Same as Fig. 7 but an alternate trajectory that has a start point at $5^{\circ} \mathrm{W}$ longitude and $55^{\circ} \mathrm{N}$ latitude.

Although the error in the estimated $\beta$ and $A$ might be large at any specific timestep, the three trajectory examples demonstrate that the time history can be adequately recreated using the parameterized effective radius and number density. This has the potential to be very useful in comparing a bulk PMC parameterized GCCM to satellite PMC radiance measurements such as those taken on the AIM satellite.

\subsection{Comparison with AIM/SOFIE}

The Solar Occultation for Ice Experiment (SOFIE) on the AIM satellite uses the technique of satellite solar occulta- tion to measure vertical profiles of limb path atmospheric transmission (Hervig et al., 2009). Their measurement technique allows for the first reported independent concurrent altitude profiles of PMC effective radius, $i w c$ and temperature from a satellite. To validate the parameterization we use all the combinations of $r_{e}, i w c$, and $T$ above $79 \mathrm{~km}$ for SOFIE's 2007 northern hemisphere season (Data Version 1.022d), similar to that described in Sect. 2, Fig. 3a for the ensemble of CARMA simulations. Figure 9a illustrates the surface plot of the relationship of these parameters as measured from SOFIE. While the absolute values are slightly different (SOFIE temperatures are slightly warmer and $i w c$ 

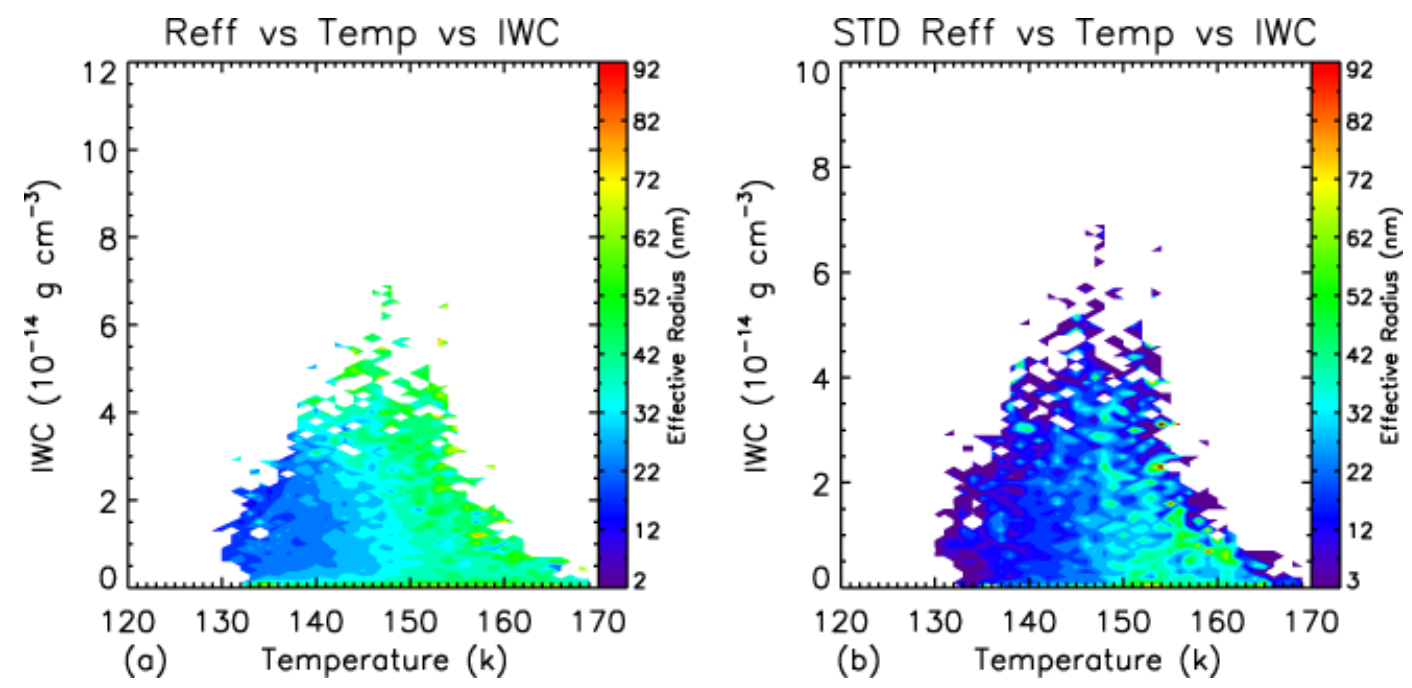

Fig. 9. Surface plot of SOFIE data showing the relationship between $T$, $i w c$ and effective radius.

slightly less than the CARMA values shown in Fig. 3) the relationship between the variables is very similar to that determined by the CARMA simulations. Figure 9a, like Fig. 3a, illustrates that for a given $i w c$, effective radius increases with increasing temperature. Figure $9 \mathrm{~b}$ shows the standard deviation of SOFIE effective radius. It has the same general shape as Fig. 3b, where the particles are very small with not much variation at the cold extreme temperatures. The particles are bigger and mixed at the warmer temperatures with a standard deviation around $15-20 \mathrm{~nm}$. Since SOFIE data is obtained from concurrent altitude profiles of temperature, $i w c$ and effective radius, the inherent relationship of the background temperature to the effective radius distribution is upheld. Figure 9 demonstrates that the compact relationship between these variables is observed in SOFIE PMC measurements and validates the parameterization. Although not shown here, all four available seasons of SOFIE data (NH and SH 2007, NH and SH 2008) were analyzed. All seasons demonstrated the same stable relationship as that illustrated in Fig. 9.

We compare the effective radius particle distribution calculated from the CARMA simulations to the effective radius particle distribution measured from SOFIE to further validate the modeled distribution. In addition we compare to the effective radius distribution measured from a lidar at ALOMAR. Figure 10 illustrates the comparison. The black and blue curves are identical to those shown in Fig. 5 for CARMA. The red curve shows the distribution of SOFIE effective radius at all altitudes for the whole 2007 northern hemisphere season. All effective radii above $79 \mathrm{~km}$ are used, as advised in Hervig et al. (2009). The green curve shows the effective radii measured from the ALOMAR lidar $\left(69^{\circ} \mathrm{N}\right)$ during the 2007 summer season (Baumgarten et al., 2008; Hervig et al., 2009). At first glance, the distributions might appear dissimilar, however there is excellent agreement of

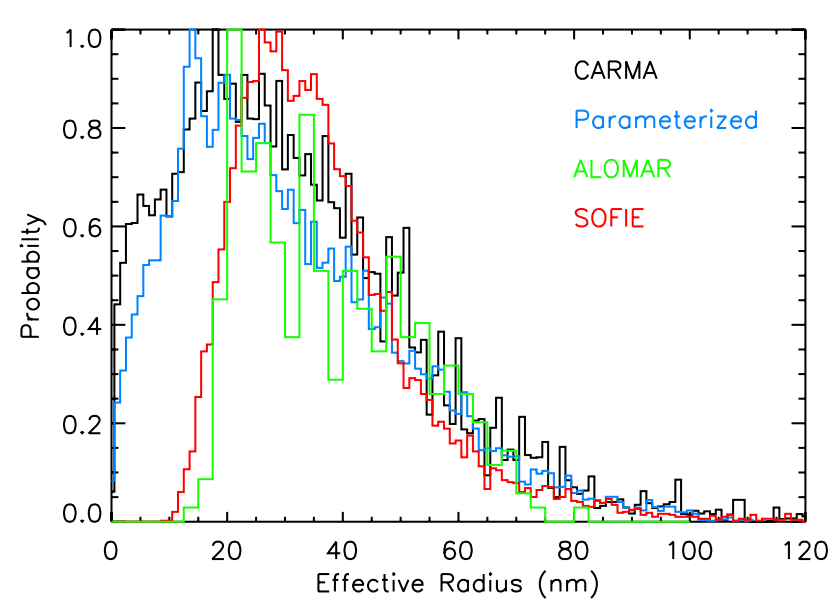

Fig. 10. Histogram of effective radius from recent satellite and ground-based measurements compared to particle distribution used to define parameterization. SOFIE NH 2007 measurements are depicted by the red line and ALOMAR lidar observations are depicted by the green line.

the distributions above $40 \mathrm{~nm}$. In this particle size region, observational techniques have the least error in measuring PMC particle size. Below $40 \mathrm{~nm}$, the distributions slightly diverge. At the smallest particle range CARMA predicts that most of the particles lie in the $10-30 \mathrm{~nm}$ range, while the SOFIE and ALOMAR distributions drop off (Hervig et al., 2009). This is not surprising since the CARMA distribution is not limited by an observing technique and it is in this particle size range that observations are the least sensitive to the true particle size. Although SOFIE and the lidar measures particles as small as $10 \mathrm{~nm}$, they can rarely detect the most tenuous clouds, which helps explain the absences of large numbers of small particles. It is important to also note that particle size 


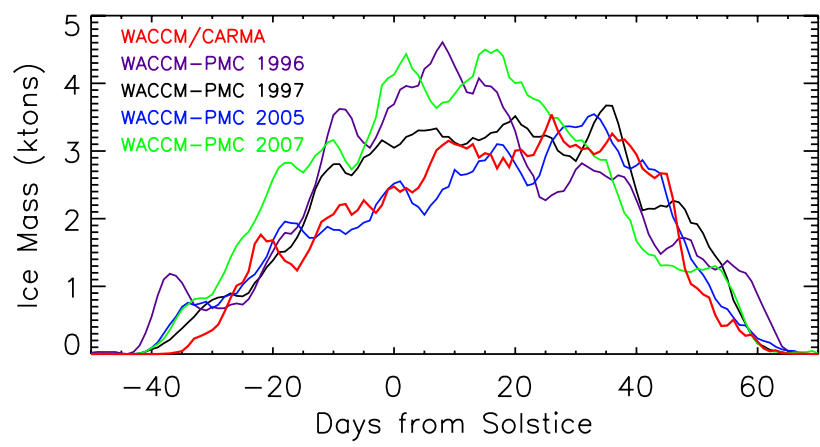

Fig. 11. Total PMC ice mass versus day from solstice. The red curve represents the total ice mass from one year of WACCM/CARMA at solar minimum conditions (Bardeen et al., 2009). The purple, black, blue and green curves represent the total ice mass from four different solar minimum years from WACCMPMC.

is only determined from SOFIE when the extinction channel $\beta(1.037 \mu \mathrm{m})$ is above the noise, which corresponds to $48 \%$ of the observations with $Z \max >79 \mathrm{~km}$ (Hervig et al., 2009). Hervig et al. (2009) explains that ice mass density is often identified in the IR measurements $\beta(3.064 \mu \mathrm{m})$ when no signal appears at $1.037 \mu \mathrm{m}$. This effect can limit the altitude range where particle sizes can be inferred, and may be preferential to the detection of larger $r_{\text {eff }}$. This may help explain the differing peaks of the distribution. Although, all the distributions have slightly different peaks, the ensemble from CARMA seems to characterize the full range of ice particle size and agrees very well with the observations from the middle to largest particles sizes. It is important that the larger particles are estimated accurately in a global model, since these dominate the optical signal and contain most of the ice mass.

\section{Implementation in the WACCM Model}

While the empirical formula that characterizes the relationship between iwc, $T$ and $r_{\text {eff }}$ can be used for a number of possible applications (i.e. estimating $T$ from satellite measurements of $r_{\text {eff }}$ and $i w c$ ), our purpose is to use it to incorporate a bulk PMC parameterization into WACCM to run decadal scale PMC simulations. While a detailed description of how we incorporated the bulk parameterization into WACCM (Marsh et al., 2007) is beyond the scope of this paper, we briefly describe the process and show a comparison to PMC data from the AIM satellite to illustrate its potential use. The version of WACCM used in this study has an enhanced vertical resolution in the mesosphere-lower thermosphere region (grid spacing $\sim 0.3 \mathrm{~km}$ ) and $2^{\circ} \times 2.5^{\circ}$ horizontal resolution. We refer to this version as WACCM-PMC.

There are many ways one could implement a nucleation process within a global model, which combined with the empirical relationship described in this paper, could be the basis of a PMC bulk parameterization. For example, one can take a simple approach whereby a very small percentage of the available water is converted to ice when the air becomes extremely super saturated. Another approach could be to calculate the dust distribution and use that as a limit on the PMC formation rate. Yet another example could be to assume large sulphate particles as a source (Mills et al., 2005) and track their density to limit the initial ice formation. For our purposes we implemented the first nucleation approach into WACCM as a "bulk" nucleation parameterization and convert $0.001 \%$ /hour of background water vapor into ice when the saturation ratio exceeds 30 .

When the saturation ratio exceeds $30, i w c$ is created based on the rate of background water vapor converted to ice. At each subsequent timestep, we calculate the effective ice particle radius $\left(r_{\mathrm{effp}}\right)$ using the local $i w c$ and background $T$, and Eq. (2). The parameterized effective radius is used to calculate the sedimentation rate and the growth rate $(\partial r / \partial t)$ of the particle. The change in the ice mass $\left(Q_{\text {growth }}\right)$ is defined by,

$Q_{\text {growth }}=A_{\text {ice }} \rho_{\text {ice }}(\partial r / \partial t)$

where, $A_{\text {ice }}$ is the total surface area of the particle, $\rho_{\text {ice }}$ is the density of ice $\left(0.93 \mathrm{~g} \mathrm{~cm}^{-3}\right), \partial r / \partial t$ is the radius growth rate defined by Gadsden (1998) and Hesstvedt (1969). The growth rate depends on the saturation ratio, background $T$ and $P_{\text {sat }}$ (the pressure of water vapor over ice). The total surface area $\left(A_{\text {ice }}\right)$ of the ice particle is:

$A_{\text {ice }}=N_{\text {para }} 4 \pi r_{\text {effp }}^{2}$

where the total number density ( $\left.N_{\text {para }}\right)$ is calculated using Eq. (3). Using the parameterized radius, $Q_{\text {growth }}$ modifies the $i w c$ carried to the next time step. At the next time step the process repeats itself, a $r_{\text {effp }}$ is determined from the $i w c$ (determined from the previous time step) and local $T$ and $Q_{\text {growth }}$ modifies the $i w c$. This is a very cost effective process since numerous particle size bins are not stored at each time step, however the calculated $r_{\mathrm{eff} p}$ are stored in the WACCM-PMC output files. WACCM-PMC model runs provide $\mathrm{PMC}$ products such as ice mass, particle effective radius, and ice number density on a global scale at any specified time step. WACCM-PMC was run for 30 consecutive years to study the PMC historical record. The results of this study will be shown in a future publication. However, to show the effectiveness of the parameterized PMC module we compare the total ice mass from $50^{\circ}-90^{\circ} \mathrm{N}$ latitude over a PMC season to that calculated using WACCM/CARMA (Fig. 11) (Bardeen et al., 2009). WACCM/CARMA is a version of WACCM where CARMA is an incorporated sectional microphysics regime to calculate PMC properties. The two techniques represent independent methods of calculating PMC properties in a 3-D GCCM. The red curve in Fig. 11 shows the total ice mass for a solar minimum year from a 

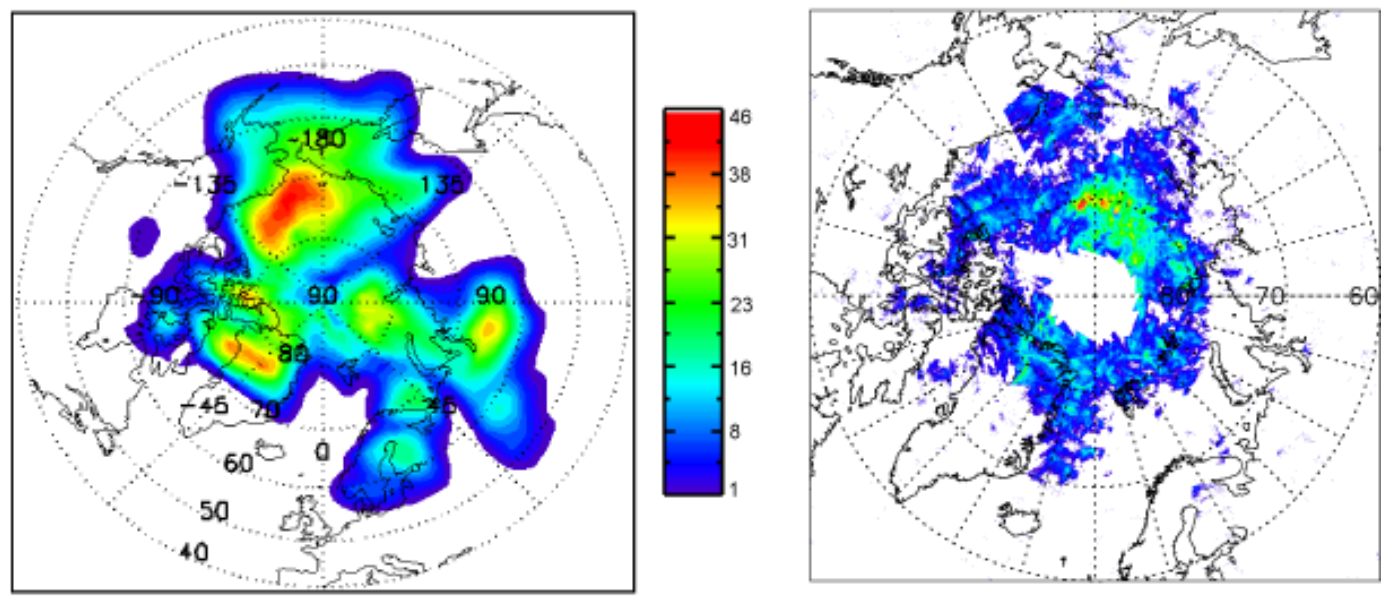

Fig. 12. PMC albedo at $265 \mathrm{~nm}$ wavelength in $1 \times 10^{-6} \mathrm{str}^{-1}$ albedo units. The left globe illustrates the WACCM PMC albedo for 21 June 2007 at 00:00 UT. The right globe shows the PMC albedo from the CIPS instrument at 12:00 local time 21 June 2007.

WACCM/CARMA simulation with similar gravity wave tunings as WACCM-PMC. This curve is shown in Fig. 13 from Bardeen et al. (2009). The 4 other curves represent solar minimum years from the 30-year WACCM-PMC run. Considering that WACCM is a free running model and that the PMC properties are solved using different methods, the total ice mass agrees very well. This demonstrates that this approach produces reasonably accurate PMC ice mass in comparison to a bin-microphysics approach.

From the PMC products in WACCM-PMC a cloud albedo and frequency can be calculated to compare to satellite observations such as the Cloud Imaging and Particle Size (CIPS) experiment on the AIM mission. An example of this type of comparison is shown in Fig. 12. Figure 12a shows a global map of PMC albedo calculated from a daily snapshot (00:00 UT) of a WACCM-PMC run. The cloud albedo is calculated using Eqs. (4), (5) and (6) at a scattering angle of $90^{\circ}$ at $265 \mathrm{~nm}$. In comparison, Fig. 12b shows the detected PMC albedo, the ratio of atmospheric radiance to solar irradiance in units of $1 \times 10^{-6} \mathrm{sr}^{-1}$ at $90^{\circ}$ scattering angle, from one day of CIPS data at 1200 local time (Bailey et al., 2009; McClintock et al., 2009; Rusch et al., 2009). It is noted that the latitude range in Fig. 12a and b are different. Because the CIPS signal is dominated by the background Rayleigh scattering at latitudes below $65^{\circ}$, detecting PMCs below this latitude is difficult (Bailey et al. 2009). Therefore the latitude range only extends to $60^{\circ}$ latitude in Fig. $12 \mathrm{~b}$. The albedo calculated from WACCM-PMC (Fig. 12a) shows cloud detections well below $65^{\circ}$ latitude because CIPS detection biases are not incorporated into the model albedo calculation. Since WACCM-PMC is a free running model the daily snapshot shown will indeed be different; however the global distribution of PMC occurrence and albedo match considerably well (above $65^{\circ}$ latitude), giving confidence in the parameterization approach. In addition, this figure shows that a parameter- ized effective radius can be used to successfully estimate the optical properties of PMCs, and demonstrates the potential of a bulk PMC parameterization in a multi-dimension global climate model.

\section{Summary and conclusions}

The means of including a representation of PMC microphysics in a multidimensional global climate model without using a Lagrangian or sectional microphysics code framework has been developed. This bulk PMC parameterization allows for theoretical ice cloud microphysics to be applied to ice particles to simulate growth, sublimation and sedimentation. This is accomplished by characterizing the relationship of the PMC effective radius to the local $T$ and $i w c$ through an empirical formula. The relationship was characterized using an ensemble of 1-D CARMA simulations forced by temperature histories produced from the WACCM model. The empirical formula is stable and is able to describe the fundamental characteristics of PMC particle growth and evolution in terms of $T$ and $i w c$, without keeping track of the time history of each ice particle or particle size bin. By adequately estimating the altitude distribution of effective radius at each grid point, it allows for an estimate of the observed optical properties of PMCs to be compared to satellite and groundbased measurements. The proposed bulk parameterization that includes a parameterization of $r_{\text {eff }}$ has the potential to be used in any multi-dimensional climate model to investigate the drivers of PMC global variability without extensive computational costs. The empirical formula has the potential to be a very powerful tool in helping to characterize satellite and ground based observations of PMCs.

PMC modeling studies that include a Lagrangian work frame such as the COMMA model (Berger and Lübken, 2006; Lübken and Berger, 2007) show that PMC particles 
have a complicated history of variation. They nucleate, grow, sublimate, advect, and are transported all over the pole on relatively short time scales. The empirical formula described here does a good job of estimating the instantaneous particle size without modeling the particle history because of the stable relationship between $i w c, T$ and effective radius that PMCs seem to encompass as shown in Figs. 3 and 9. The history of the ice particle seems to be contained in the instantaneous $T$ and $i w c$ at any given time step. For example, if the local temperature is relatively warm, but the local ice mass is relatively high, it is likely the air parcel trajectory passed through a region of cooler temperatures that led to significant particle growth and a large effective radius. The empirical formula does not directly say anything about the background water vapor. However, the background water vapor is tied into the nucleation and the growth process. In CARMA, the nucleation process puts a certain amount of the background water into solid state based on the nucleation rate. Then as CARMA progresses, it builds on that initial particle, or set of particles by converting the background water vapor to ice in the growth process. In WACCM-PMC, the key prognostic variables are water vapor, liquid and ice. The model does not attempt to transport a distribution of particles as is done in WACCM/CARMA (Bardeen et al., 2009). Therefore, it needs to only calculate the transfer of water between its phases. This is done by calculation of a growth/sublimation rate that depends on $r_{\text {eff. }}$. The amount of total water is "built in" to the $i w c$, since for a given temperature, $i w c$ will depend directly upon the total available water vapor. Comparisons of the parameter space from CARMA simulations used to calculate the effective radius parameterization with that of SOFIE, shows that the relationship between these variables, is also evident in PMC measurements. The fact that the SOFIE measurements exhibit the same relationship between these variables gives confidence in the CARMA model simulations and the robustness of the empirical formula. The compact relationship between these variables could possibly be used to help interpret observations of PMC ice mass, radius and mesospheric temperature in past and future experiments.

The bulk parameterization has been included into WACCM and produces reasonable ice mass, particle radius, and ice number density distributions on a global scale.

The details of the implementation in WACCM will be described in a future publication. Initial comparison of output from the WACCM-PMC model shows that it is comparable to the CIPS measurements from AIM. We utilized this parameterization to support a long-term run to study climate change and its effect on cloud presence. The solar minimum years show very comparable total ice mass to that calculated with WACCM/CARMA. These modeling efforts are crucial to understanding how AIM PMC observations relate to longer-term changes resulting from solar and anthropogenic forcing.
Acknowledgements. This research was supported by NASA grant \#05-SRT05-70 conducted at the National Center for Atmospheric Research. The National Center for Atmospheric Research is sponsored by the National Science Foundation. Any opinions, findings and conclusions or recommendations expressed in the publication are those of the author(s) and do not necessarily reflect the views of the National Science Foundation. Thanks to the AIM team for providing CIPS and SOFIE data. Thanks to Dave Rusch, Chuck Bardeen, Mark Hervig and Gerd Baumgarten for collaboration. Special thanks to Gary Thomas for his help and insight.

Edited by: F.-J. Lübken

\section{References}

Bailey, S. M., Thomas, G. E., Rusch, D. W., Merkel, A. W., Jeppesen, C., Carstens, J. N., Randall, C. E., McClintock, W. E., and Russell III, J. M.: Phase Functions of Polar Mesospheric Cloud Ice as Observed by the CIPS Instrument on the AIM Satellite, J. Atmos. Solar-Terr. Phys., 71, 373-380, 2009.

Bardeen, C. G, Toon, O. B., Jensen, E. J., Hervig, M. E., Randall C. E., Benze, S., Marsh, D. R., and Merkel, A. W.: Numerical simulations of the three-dimensional distribution of polar mesospheric clouds and comparisons with CIPS and SOFIE observations, J. Geophys. Res., in review, 2009.

Bardeen, C. G., Toon, O. B., Jensen, E. J., Marsh, D. R., and Harvey, V. L.: Numerical simulations off the three- dimensional distribution of meteoric dust in the mesosphere and upper stratosphere, J. Geophys. Res., 113, D17202, doi:10.1029/2007JD009515, 2008.

Baumgarten, G., Fiedler, J., Lübken, F.-J., and von Cossart, G.: Particle properties and water content of noctilucent clouds and their interannual variation, J. Geophys. Res., 113, D06203, doi:10.1029/2007JD008884, 2008.

Berger, U. and Lübken, F.-J.: Weather in mesospheric ice layers, Geophys. Res. Lett., 33, L04806, doi:10.1029/2005GL024841, 2006.

Bohren, C. F. and Huffman, D. R.: Absorption and Scattering of Light by Small Particles Wiley-Interscience, New York, 1983.

Boudala, F. S., Isaac, G. A., Fu, Q., and Cober, S. G.: Parameterization of ice particle sizes for high latitude ice clouds, Int. J. Climate., 22, 1267-1284, 2002.

Chandran, A., Rusch, D. W., Palo, S. E., Thomas, G. E., and Taylor, M.: Gravity wave observations in the summertime polar mesosphere from the cloud imaging and particle size (CIPS) experiment on the AIM spacecraft, J. Atmos. Sol.-Terr. Phys., 71, 392400, 2009.

DeLand, M. T., Shettle, E. P., Thomas, G. E., and Olivero, J. J.: Latitude-dependent long-term variations in polar mesospheric clouds from SBUV version 3 PMC data, J. Geophys. Res., 112, D10315, doi:10.1029/2006JD007857, 2007.

Gadsden, M.: The north-west Europe data on noctilucent clouds: A survey, J. Atmos. Sol. Terr. Phys., 60, 1163-1174, 1998.

Garcia, R. R., Marsh, D. R., Kinnison, D. E., Boville, B. A., and Sassi, F.: Simulation of secular trends in the middle atmosphere, 1950-2003, J. Geophys. Res., 112, D09301, doi:10.1029/2006JD007485, 2007.

Hervig, M. E., Gordley L. L., Stevens, M. H., Russell III, J. M., Bailey, S. M., and Baumgarten, G.: Interpretation of SOFIE PMC 
measurements: Cloud identification and derivation of mass density, particle shape and particle size, J. Atmos. Solar-Terr. Phys., 71, 316-330, doi:10.1016/j.jastp.2008.07.009, 2009.

Hesstvedt, E.: The physics of nacreous and noctilucent clouds, in: stratospheric circulation, edited by: Webb, W. L., 4, 207-217, London \& New York: Academic, 1969.

Hunten, D. M., Turco, R. P., and Toon, O. B.: Smoke and dust particles of meteoric origin in the mesosphere and stratosphere, J. Atmos. Sci., 37, 1342-1357, 1980.

Karlsson, B. and Rapp, M.: Latitudinal dependence of noctilucent cloud growth, Geophys. Res. Lett., 33, L11812, doi:10.1029/2006GL025805, 2006.

Lübken, F.-J. and Berger, U.: Interhemispheric comparison of mesospheric ice layers from the LIMA model, J. Atmos. Sol., Terr. Phys., 69, 17-18, 2007.

Marsh, D. R., Merkel, A. W., Gettelman, A., Bardeen, C. G., Rusch, D. W., and Hervig, M. E.: Simulation of Polar Mesospheric clouds within a 3-Dimensional Chemistry Climage Model, EOS Trans. AGU, 88(52), Fall Meet. Suppl., Abstact SA13B-07, 2007.

McClintock, W. E., Rusch, D. W., Thomas, G. E., Merkel, A. W., Lankton, M. R., Drake, V. A., Bailey, S. M., and Russell III, J. M.: The Cloud Imaging and Particle Size Experiment On The Aeronomy Of Ice In The Mesosphere Mission: Instrument Concept, Design, Calibration, And On-Orbit Performance, J. Atmos. Solar-Terr. Phys., 71, 340-355, 2009.

Merkel, A. W., Rusch, D. W., Palo, S. E., Russell III, J. M., and Bailey, S. M.: Mesospheric planetary wave effects on global PMC variability inferred from AIM-CIPS and TIMED-SABER for the northern summer 2007 PMC season, J. Atmos. Solar-Terr. Phys., 71, 381-391, doi:10.1016/j.jastp.2008.12.001, 2009.
Mills, M. J., Toon, O. B., and Thomas, G. E.: The mesospheric sulfate aerosol layer, J. Geophys. Res., 110, D24208, doi:10.1029/2005JD006242, 2005.

Rapp, M. and Thomas, G. E.: Modeling the microphysics of mesospheric ice particles: assessment of current capabilities and basic sensitivities, J. Atmos. Sol. Terr. Phys., 68, 715-744, 2006.

Rusch, D. W., Thomas, G. E., McClintock, W., Merkel, A. W., Bailey, S. M., Russell III, J. M., Randall, C. E., Jeppesen, C., and Callan, M.: The Cloud Imaging and Particle Size Experiment on the Aeronomy of Ice in the Mesosphere Mission: Cloud Morphology for the Northern 2007 season, J. Atmos. Solar-Terr. Phys., 71, 356-364, doi:10.1016/j.jastp.2008.11.005, 2009.

Russell III, J. M., Bailey, S. M, Horanyi, M., Gordley, L. L., Rusch, D. W., Hervig, M. E., Thomas, G. E., Randall, C. E., Siskind, D. E., Stevens, M. H., Summers, M. E., Taylor, M. I., Englert, C. R., Espy, P. J., McClintock, W. E., and Merkel, A. W.: Aeronomy of Ice in the Mesosphere (AIM): Overview and early science results, J. Atmos. Solar-Terr. Phys., 71, 289-299, 2009.

Siskind, D. E. and Stevens, M. H.: A radiative feedback from an interactive polar mesospheric cloud parameterization in a two dimensional model, Adv. Space Res., 38, 2383-3287, 2006.

Shettle, E. P., DeLand, M. T., Thomas, G. E., and Olivero, J. $\mathrm{J}$.: Long term variations in the frequency of polar mesospheric clouds in the Northern Hemisphere from SBUV, Geophys. Res. Lett., 36, L02803, doi:10.1029/2008GL036048, 2009.

Thomas, G. E.: Are noctilucent clouds harbingers of global change in the middle atmosphere?, Adv. Space Res., 32(9), 1737-1746, 2003.

Wyser, K.: The effective radius in ice clouds, J. Climate, 11, 1793$1802,1998$. 